\title{
Difficult Questions and Solutions of Engineering Cost
}

\author{
Jinbao Cao ${ }^{1, ~ a ~, ~ Y u n z h u ~ L i u ~}{ }^{1, b}$, Jian Tang ${ }^{1, c}$ \\ Nanchang Institute of Technology, Nanchang 330044, China

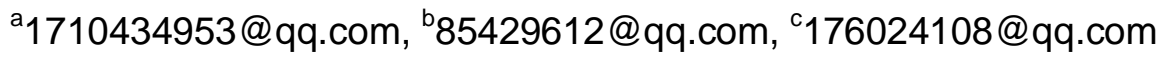

\begin{abstract}
Key Words: engineering cost; analysis and solution; management system; management method Abstract: with the social progress and economic development, the construction industry in China has also yielded rapid development in recent years, and gradually become an important pillar of Chinese national economy. However, with the continuous expansion of the construction industry in scale, the questions existing in this industry have become increasingly prominent. Specifically speaking, the questions existing in engineering cost are the most apparent. At present, the questions existing in the engineering cost in China are mainly the backward philosophy and methods of management, and the imperfect management system, etc. Reinforcing the analysis of these questions existing in the engineering cost in China and further intensifying the research and discussion of relevant solutions is highly necessary to the further promotion of the sustainable and stable development of the Chinese construction industry. In this paper, the questions existing in the engineering cost of the construction industry in China are studied and analyzed, and relevant solutions are further put forward. It is hoped that the current engineering cost of the construction industry in China can be effectively improved, the economic benefit of the construction industry can be enhanced, and the long-term development of this industry can be further facilitated in China.
\end{abstract}

\section{Introduction}

With the continuous development and expansion of the real estate cause, Chinese architectural technology has yielded certain progress. In the meanwhile, enterprises specialized on construction have also been confronted with greater questions and challenges. Although the architecture cause has yielded certain progress and achievement in the long-term development in China, there's still a huge gap from some advanced Western countries. For instance, the serious waste of construction resources and unstable control of engineering cost still exist in the construction industry in China. At present, the engineering cost in China hasn't been consistently regulated. Not only has this brought certain adverse influence to the engineering cost, but also it has been a main cause hindering the further development of Chinese construction industry. As market competition becomes gradually aggravated, the construction industry must reinforce the management of the engineering cost, strive to reduce the cost of construction production, raise the economic benefit of construction companies and guarantee the quality of architectural engineering if it wants further development. Hence, the questions existing in the engineering cost of the construction industry in China are comprehensively analyzed. Furthermore, relevant solutions are further proposed.

\section{Questions Existing in Engineering Cost}

False, incontrollable and distorted engineering cost is the main question in the present stage. Also, these questions are also common in the engineering cost in China. There are many causes to the above-mentioned questions in the engineering cost. A lot of decision-makings are involved in engineering construction, and each link in each decision-making stage is closely related with the overall construction project. Hence, if the decision-makings in construction are unscientific or unreasonable, certain contradictions will be generated in the fund control and investment in construction. Consequently, they will radically end up with the overall construction being out of control.

Moreover, construction units will also suppress the engineering cost to a certain extent. This severely violates the law of value in the market, because the extreme suppression of the engineering 
cost will lead to an extremely low cost, which will cause a significant difference between the successful bidders' price and the engineering cost. On the other hand, construction units are so desperate to win the bidding in the fierce competition that they intend to control the engineering cost. As a result, the engineering cost will be extremely low. Hence, in order to meet the standard of the engineering cost, the construction units often "cheat on workmanship and materials". For instance, they cut down the procedure of construction, and select some cheap and inferior materials in the procurement, etc.

Other than this, changes of the construction scheme will also exert certain influence on the construction to a certain extent. This is mainly occurred in the construction stage. Any alternation of the construction scheme during the construction stage will increase the engineering cost. The construction should be equipped with professionals specialized on the engineering cost, so that they can supervise the whole process. Provided that the practical construction is inconsistent with the design scheme, new items will be added, and the original engineering cost will no longer be applicable to the practical project. Thus, the engineering cost needs to be evaluated again, which will lead to extra cost. Hence, a vicious cycle which involves uncontrollable, distorted and deflected engineering cost will be generated and exert serious influence on the overall construction.

\section{Questions in Information Management of Engineering Cost}

At present, information management is an important content of corporate management. With the continuous development of science and technology, the existing information technology has been extensively applied in all circles of the society. In the meanwhile, enterprises' capability in information management has also been improving. While bringing new opportunities to the various industries, economic integration also brings certain challenges to their development. Under the new market pattern, the construction industry must march towards the direction of information-oriented management if it wants to yield further development. Only by achieving these can it satisfy the demands of the information-oriented era and become much more scientific and reasonably. The rapid development of the construction industry puts forward brand-new requirements towards the industry. In order to get adapted to the development demands of the construction industry, the engineering cost must be scientific, intelligent and reasonable. That's exactly the direction of engineering cost reform proposed by the information era.

Nowadays, information technology has been promoted and applied in the engineering cost to a certain extent. It is mainly reflected in the application of the various types of engineering cost software. Also, this symbolizes another big step in the engineering cost in China. With the continuous increase of information platforms and services on the engineering cost, questions existing in the information management of the engineering cost in China have become increasingly prominent. These questions include, but are not limited to the narrow scope of information collection, the backward philosophy of management and the lack of experience in management, etc.

Backward means of information collection is the most prominent question in the information management of the engineering cost. Not only will it exert certain influence on the authenticity of the engineering cost, but also it will play an adverse role in the management of the engineering cost. Since the information classification of the engineering cost is relatively disordered, different professionals in the same project will provide different results of the engineering cost through their respective analysis. In the meanwhile, the backward means of information collection has also severely affected the authenticity and validity of the engineering cost.

Furthermore, the deficiency of the funds for investment management is also a main question influencing the quality and efficiency of the information management. The deficiency of funds for information management will lead to the failure of attracting excellent professionals. Hence, there will be a shortage of talents, which will lead to the increasing workload on every existing professional. Also, the increasing workload will easily lead to the increase of errors in the daily work. On the other hand, the deficiency of the management funds will also result in old-fashioned and backward information management, which will further affect the correctness and accuracy of the engineering cost. To a certain extent, it will also lead to the increase of the construction cost, and 
further lead to the increase of the overall engineering cost and affect the economic benefit that the construction company can obtain.

\section{Solutions to Engineering Cost Management}

Through the above research and analysis, we can clearly cognize the questions existing in the engineering cost and analyze their influence on the engineering cost. Hence, it is highly necessary to adopt certain means to renovate and improve such a dilemma in the engineering cost in China.

First of all, the management of the construction projects should be reinforced to guarantee that the engineering cost is authentic, scientific and reasonable. Besides, the questions existing in the engineering cost should be further studied and analyzed, so as to locate the basic causes to these questions. And then, relevant measures and solutions can be proposed for further improvement. Since each stage of the implementation is closely related with the overall quality of the construction, solving the questions underlying in the engineering cost, and meticulously studying and analyzing each link of the construction is highly necessary. Guaranteeing the validity and reasonability of each construction stage can further ensure the overall quality of the construction. The management strategies of the engineering cost exert a critical influence on the efficiency and quality of the overall engineering cost. Hence, in order to effectively alleviate the influence on the engineering cost brought by the questions existing in the cost management strategies, the demands of the constructional engineering should be taken as the starting point, the investment of funds should be reinforced and the cultivation of talents should be emphasized. In the meanwhile, professionals specialized on the engineering cost should be employed to supervise the construction in real time, so that the influence on the engineering cost brought by engineering faults can be avoided.

Secondly, the bidding work is a key in the management of the engineering cost. In order to effectively guarantee the normal progress of the bidding work, the construction units should form a comprehensive cognition of the practical demands of architectural engineering ideologically. In the meanwhile, the construction units should further clarify the practical demands of the architectural engineering. The bidding work will directly affect the implementation of the overall project, and further exert certain influence on the engineering cost. Hence, the bidding work should strictly comply with the procedure, so that it can be guaranteed to be authentic and scientific. In the meanwhile, scientific and reasonable bidding schemes should be formulated for the bidding work, and any blind bidding should by no means be allowed. Moreover, the characteristics and the actual demands of implementation should be taken into consideration in the bidding schemes. The bidding price should be reasonably controlled, so that the engineering construction can be completed with guaranteed quality and quantity.

Any alternation to the construction schemes during the construction process will lead to severe influence on the engineering cost. Hence, it is highly necessary to reinforce the management of cost schemes during the construction. Bearing the objective of effectively preventing the construction side from altering the construction schemes arbitrarily, the schedule of construction should be taken into full consideration. In the meanwhile, the construction side should analyze the characteristics of architectural engineering, guarantee the schedule of construction, and effectively avoid prolonging or excessively shortening the scheduled construction period.

The backward philosophy of the engineering cost management is the core factor influencing the engineering cost. Hence, it is very necessary to update the philosophy of such management in time. Since there's a huge gap between the management philosophy of the engineering cost in the traditional sense and under the background of the new era, the management of the engineering cost through the traditional philosophy fails to keep pace with the development of the construction industry. The philosophy of information-oriented management is a new philosophy of management in the engineering cost. The management of information-oriented engineering cost can effectively improve the collection and classification of valuable information, and further guarantee the accuracy and reasonability of the engineering cost. Only by achieving these can the normal operation of the construction be facilitated. Since architectural engineering involves relatively extensive information, the scope of information collection should be properly extended, so that the 
comprehensiveness of information can be guaranteed. Only by guaranteeing the comprehensiveness of information collection can the accuracy of the engineering cost be further raised.

Finally, it is also imperative to effectively classify information in architectural engineering. At present, the relatively disordered information classification in architectural engineering has brought extreme difficulties to the engineering cost. Hence, in order to effectively avoid the negative influence brought by the disordered information on the engineering cost, the information management system of the engineering cost should be further standardized and unified. Furthermore, the information concerning architectural engineering should be rigorously classified and filed.

\section{Conclusion}

It is suggested by the analysis above that many questions exist in the engineering cost in China, and they have severely affected the quality of the engineering cost. Also, these questions have further influenced the development of the construction industry in China. Therefore, the questions existing in the engineering cost in China are mainly analyzed, and the causes and influence of these questions are profoundly studied. Finally, in light of the questions existing in the engineering cost, relevant solutions are proposed. It is hoped that the questions existing in the engineering cost in China can be effectively improved, and the sustainable and stable development of the construction industry can be further facilitated. Furthermore, the residents' dwelling quality can also be improved simultaneously.

\section{References:}

[1] Zhang Fulong. On the Budget and Settlement Auditing of Engineering Cost [J]. Charming China, 2009, (33): 109-110.

[2] Zhao Yinghua. On the Questions and Countermeasures of the Settlement of Engineering Cost [J]. Value Engineering, 2013, 32(4): 62-63.

[3] Huanglei. On the Management and Control of Engineering Cost of Civil Engineering Projects [J]. Value Engineering, 2013, 32(4): 95-96.

[4] Yuan Zhongwei. The Analysis of Questions and Measures of Modern Engineering Cost [J]. Standardization of Engineering Construction, 2014, (10):117-117.

[5] Chenqun. The Analysis of Questions Found in the Settlement Auditing of Engineering Cost [J]. China Homes, 2014, (12): 385-385.

[6] Ni Wenjuan. On the Questions and Control Measures of Engineering Cost [J]. New Material New Decoration, 2013, (12): 292-292,295.

[7] Shaorui, Zhang Jiangao. On the Questions and Tendency of Information-oriented Management Development of Engineering Cost [J]. Shanxi Architecture, 2009, 35(5): 232-233.

[8] Tian Wengang. The Current Condition and Questions of Modern Engineering Cost [J]. Technology Innovation and Application, 2013, (21): 231-231.

[9] Zhangyu. The Analysis of Common Questions and Countermeasures of Engineering Cost [J]. Urban Construction Theory Research, 2014, (11). 